\title{
Strain-attenuated spin frustration in double perovskite $\mathrm{Sr}_{2} \mathrm{FeOsO}_{6}$
}

\author{
Paresh C. Rout ${ }^{\circ}$ and Udo Schwingenschlögl* \\ Physical Sciences and Engineering Division, KAUST, Thuwal 23955-6900, Kingdom of Saudi Arabia
}

(Received 3 May 2020; revised 3 November 2020; accepted 4 January 2021; published 15 January 2021)

\begin{abstract}
Using density functional theory together with Monte Carlo simulations, we demonstrate that epitaxial strain, both compressive and tensile, attenuates the spin frustration of double perovskite $\mathrm{Sr}_{2} \mathrm{FeOsO}_{6}$ to significantly enhance the critical temperature to $310 \mathrm{~K}$, enabling room-temperature applications. We discover under tensile strain a tetragonal $(\mathrm{I} / \mathrm{m})$-to-monoclinic $\left(P 2_{1} / n\right)$ structural transition concomitant with an antiferromagnetic-toferrimagnetic transition. Furthermore, an indirect-to-direct band gap transition is observed with the valence and conduction states localized on different transition metal sublattices, opening a route to efficient electron-hole separation upon photoexcitation.
\end{abstract}

DOI: 10.1103/PhysRevB.103.024426

\section{INTRODUCTION}

Transition metal double perovskite oxides of the general formula $A_{2} B B^{\prime} \mathrm{O}_{6}$, with $A$ being an alkali, alkaline earth, or rare earth atom and $B / B^{\prime}$ being a transition metal atom, continue to attract attention because of versatile magnetic properties such as high-temperature ferrimagnetism [1], half metallicity [2-4], insulating ferro- and ferrimagnetism [5-7], large magnetoresistance [8], and large coercivity and exchange bias [9]. Particularly, strongly localized $3 d$ valence orbitals at the $B$ site and more electron-itinerant $4 d / 5 d$ valence orbitals at the $B^{\prime}$ site favor high-temperature magnetism as required for many spintronics applications.

Several $3 d-4 d$ and $3 d-5 d$ compounds with alkaline earth or rare earth atoms at the $A$ site have been synthesized experimentally using various techniques [10-18]. Among the $3 d-5 d$ compounds, $\mathrm{Ca}_{2} \mathrm{FeOsO}_{6}$ is monoclinic $\left(P 2_{1} / n\right)$ [17] and $\mathrm{Sr}_{2} \mathrm{FeOsO}_{6}$ tetragonal $(\mathrm{I} / \mathrm{m})$ [15]. $\mathrm{Sr}_{2} \mathrm{CrOsO}_{6}$ (highest critical temperature of $725 \mathrm{~K}$ among all perovskite oxides) and $\mathrm{Sr}_{2} \mathrm{CoOsO}_{6}$ undergo structural phase transitions from rhombohedral $(R \overline{3}$; at $300 \mathrm{~K})$ to cubic $(F m \overline{3} m$; at $540 \mathrm{~K})[16]$ and monoclinic $(I 2 / \mathrm{m}$; below $108 \mathrm{~K})$ to tetragonal $(\mathrm{I} / \mathrm{m}$; above 108 K) [13], respectively. Magnetic susceptibility and specific heat measurements show that $\mathrm{Ca}_{2} \mathrm{FeOsO}_{6}$ is an insulating ferrimagnet with a high critical temperature of $320 \mathrm{~K}$ [17], while $\mathrm{Sr}_{2} \mathrm{FeOsO}_{6}$ undergoes an antiferromagnetic (AFM)-toAFM transition at $67 \mathrm{~K}$ and has a critical temperature of $140 \mathrm{~K}$ [15]. According to neutron diffraction experiments, the two AFM phases of $\mathrm{Sr}_{2} \mathrm{FeOsO}_{6}$ differ from one another in the magnetic ordering along the $c$ axis but not in the $a b$ plane [19-21]. Along the $c$ axis, there is AFM coupling between ferromagnetically coupled double layers in the lowtemperature AFM phase and ferromagnetic (FM) coupling in the high-temperature AFM phase (C-AFM phase). In the C-AFM phase, FM out-of-plane superexchange arises from the $180^{\circ} \mathrm{Fe}-\mathrm{O}-\mathrm{Os}\left(3 d^{5}-2 p^{6}-5 d^{3}\right)$ bond angle along the $c$ axis

*udo.schwingenschlogl@kaust.edu.sa and AFM in-plane superexchange from the $\sim 165^{\circ} \mathrm{Fe}-\mathrm{O}$-Os bond angle in the $a b$ plane.

The low critical temperature $(140 \mathrm{~K})$ of $\mathrm{Sr}_{2} \mathrm{FeOsO}_{6}$ can be slightly enhanced by the substitution of smaller cations at the $A$ site due to the generation of small structural distortions (magnetostructural coupling) [22]. For example, when half of the $\mathrm{Sr}$ atoms are replaced with $\mathrm{Ca}$ atoms the structure becomes monoclinic and the ordering ferrimagnetic (FiM) with a critical temperature of $210 \mathrm{~K}$. On the other hand, an AFM-to-FiM transition driven by hydrostatic pressure has been reported and attributed to increasingly different cubic crystal field splittings at the Fe and Os sites, which precludes FM coupling of the $e_{g}$ electrons along the $c$ axis [23]. As a result, the AFM coupling of the $t_{2 g}$ electrons becomes dominant in both the in-plane and out-of-plane directions, leading to FiM ordering, however, only as long as the hydrostatic pressure is maintained.

A route to permanent FiM ordering and a significantly elevated critical temperature could be the application of epitaxial strain, because the observed low values recently have been attributed to spin frustration [24]. Generally, experimental progress in achieving high-quality thin films makes it possible to employ epitaxial strain for controlling the material properties. Specifically, experiments indicate that tensile strain (reduction of the $c / a$ ratio) alters the $180^{\circ}$ out-of-plane superexchange angle of $\mathrm{Sr}_{2} \mathrm{FeOsO}_{6}$ [23]. In this paper, we therefore use first-principles calculations to show that epitaxial strain is an effective tool to tune the magnetic properties of $3 d-5 d$ compounds. Particularly, we consider $\mathrm{Sr}_{2} \mathrm{FeOsO}_{6}$, as this compound can be grown on various substrates [25] and the $3 d(\mathrm{Fe})$ and $5 d(\mathrm{Os})$ atoms form a checkerboard lattice in each layer. Monte Carlo simulations are used to show that epitaxial strain, both compressive and tensile, attenuates the spin frustration of $\mathrm{Sr}_{2} \mathrm{FeOsO}_{6}$ and, hence, significantly enhances the critical temperature of the FiM phase.

\section{COMPUTATIONAL DETAILS}

We employ spin-polarized density functional theory (DFT) and the generalized gradient approximation of Perdew-Burke- 

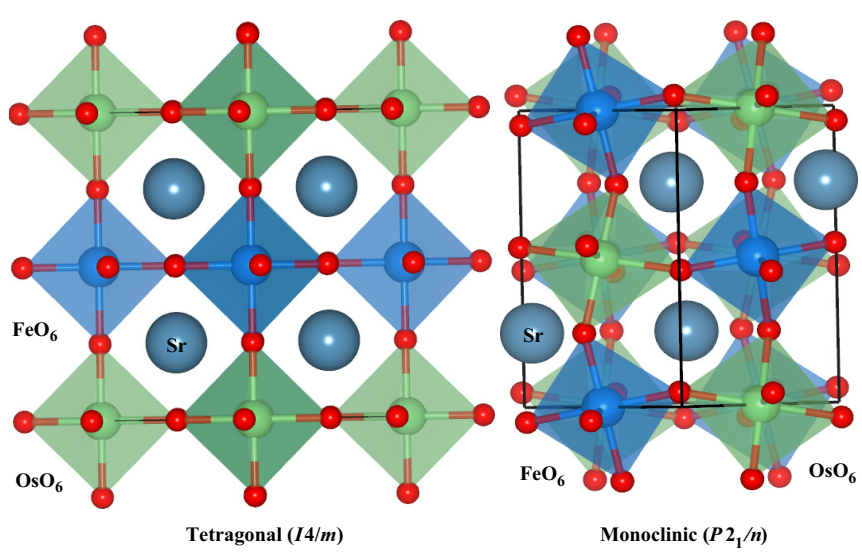

FIG. 1. Supercells with $a^{-} a^{-} c^{+}$(left; $\left.P 2_{1} / n\right)$ and $a^{0} a^{0} c^{-}$(right; $I 4 / m$ ) tilt patterns of the oxygen octahedra.

Ernzerhof for the exchange correlation functional, using the QUANTUM ESPRESSO code [26]. We account for the electron correlation effects in the $3 d$ and $5 d$ transition metal orbitals by the DFT $+U$ approach [27]. For the on-site interaction parameter we adopt the established literature values of $4 \mathrm{eV}$ (Fe) and $2 \mathrm{eV}$ (Os) [21]. Despite the presence of heavy elements, spin-orbit coupling turns out to have no impact on the electronic structure of $\mathrm{Sr}_{2} \mathrm{FeOsO}_{6}$ without strain (in agreement with Refs. [7,17,21]), except for a slight increase of the atomic magnetic moments without affecting the total magnetic moment, and therefore is neglected in calculations under strain to reduce the computational costs. Test calculations both without strain (C-AFM phase) and under $4 \%$ tensile strain (FiM phase) show that the spin-orbit coupling does not alter the crystal symmetry. A plane-wave cutoff of $90 \mathrm{Ry}$ is used in the wave-function expansion and a 640 Ry cutoff for the augmentation charge. The Brillouin zone is integrated on a $8 \times 8 \times 6$ Monkhorst-Pack $k$ mesh in the structure optimization and on a denser $14 \times 14 \times 12$ Monkhorst-Pack $k$ mesh for calculating the density of states (DOS). All structures are optimized until the Hellmann-Feynman forces stay below $0.26 \mathrm{meV} / \AA$.

Starting from the cubic structure of $\mathrm{Sr}_{2} \mathrm{FeOsO}_{6}$, we construct 20-atom $\sqrt{2} \times \sqrt{2} \times 2$ tetragonal supercells to enable magnetic ordering. By rotating the oxygen octahedra we reduce the symmetry to achieve antipolar $I 4 / m$ and $P 2_{1} / n$ space groups (see Fig. 1), which are compatible with cubic substrates $[28,29]$. Starting from the pseudocubic lattice parameter of $3.92 \AA[30]$, we mimic epitaxial strain $\epsilon$ by varying the in-plane lattice constant as $a(\epsilon)=\sqrt{2}(1+\epsilon) \times 3.92 \AA$ (limited to values that realistically can be achieved by a substrate). The out-of-plane lattice vector then is optimized (length and direction) for each value of $\epsilon$ simultaneously with the atomic positions, corresponding to (001) epitaxial growth. This procedure is executed for the magnetic orderings shown in Fig. 2, both for the $I 4 / m$ and $P 2_{1} / n$ symmetries to capture the lattice effect on the relative stabilities.

\section{RESULTS AND DISCUSSION}

The obtained energies are depicted in Fig. 3 with reference to the global minimum $\left(P 2_{1} / n\right.$ symmetry at $2 \%$ strain $)$. We

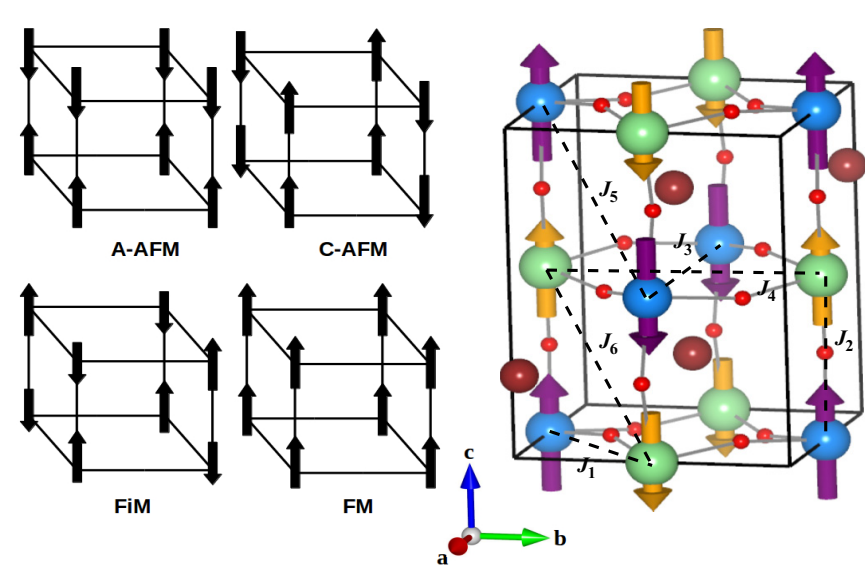

FIG. 2. Considered magnetic orderings (left) and exchange paths connecting the $\mathrm{Fe}$ and $\mathrm{Os}$ atoms (right; arrows represent the magnetic moments in the C-AFM phase).

find that the magnetic ground state changes from C-AFM to FiM ordering at $0.8 \%$ tensile strain, which increases the total magnetic moment from 0 to $2 \mu_{B}$ per formula unit. Simultaneously, the compound undergoes a structural transition from $I 4 / m$ to $P 2_{1} / n$ symmetry. Constrained to $I 4 / m$ symmetry, we observe a transition from C-AFM to FiM ordering at 2.2\% tensile strain during that the Fe-Os distance along the $c$ axis shrinks to $3.97 \AA$, in agreement with the experimental behavior under pressure along the $c$ axis [23]. Although the $\mathrm{Fe}-\mathrm{O}-\mathrm{Os}$ bond angle remains $180^{\circ}$ along the $c$ axis (favoring FM double exchange), reduction of the $c / a$ ratio by $5.8 \%$ enhances the difference between the crystal field splittings at the Fe and Os sites. This weakens the FM double exchange relative to the AFM superexchange. A transition to AFM coupling along the $c$ axis, while the in-plane coupling remains AFM due to persistent Fe-O-Os buckling, leads to FiM ordering under the $I 4 / m$ symmetry constraint.

A similar mechanism applies to the magnetic transition accompanying the transition from $I 4 / m$ to $P 2_{1} / n$ symmetry

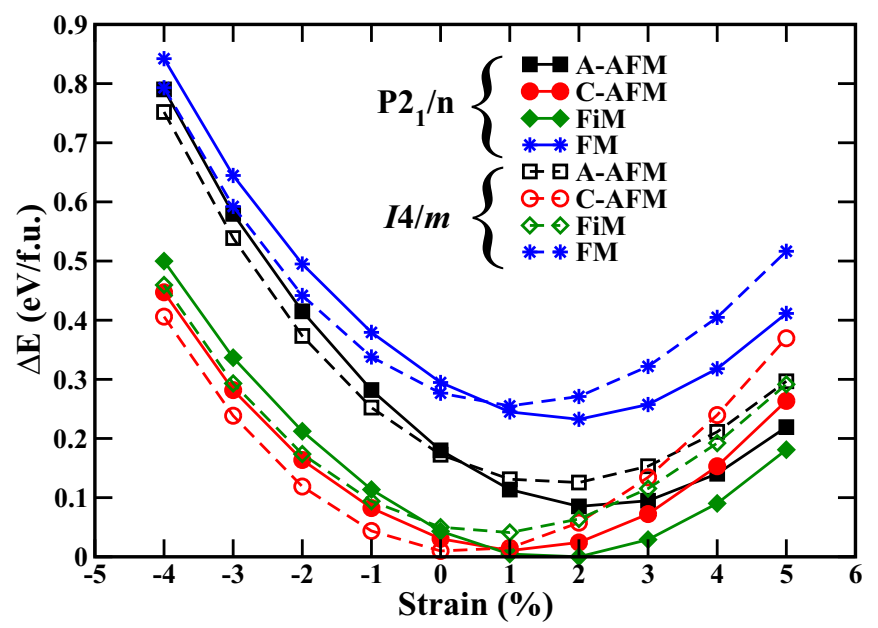

FIG. 3. Relative energies of magnetic orderings as functions of epitaxial strain for the $I 4 / m$ (dashed lines) and $P 2_{1} / n$ (solid lines) symmetries. 
TABLE I. Fe and Os magnetic moments $\left(\mu_{B}\right)$ for different strains and magnetic orderings.

\begin{tabular}{ccccccccccc}
\hline \hline & $E_{1}$ & $E_{2}$ & $E_{3}$ & $E_{4}$ & $E_{5}$ & $E_{6}$ & $E_{7}$ & $E_{8}$ & $E_{9}$ & $E_{10}$ \\
\hline$-4 \%$ & $3.93,1.27$ & $3.85,1.21$ & $3.85,1.20$ & $3.94,1.25$ & $3.85,1.18$ & $3.85,1.23$ & $3.85,1.18$ & $3.85,1.25$ & $3.85,1.18$ & $3.85,1.25$ \\
$-3 \%$ & $3.96,1.29$ & $3.88,1.23$ & $3.89,1.22$ & $3.97,1.38$ & $3.89,1.19$ & $3.88,1.26$ & $3.88,1.20$ & $3.88,1.27$ & $3.88,1.20$ & $3.88,1.27$ \\
$-2 \%$ & $3.98,1.31$ & $3.92,1.24$ & $3.92,1.24$ & $3.99,1.39$ & $3.92,1.20$ & $3.91,1.27$ & $3.90,1.24$ & $3.90,1.27$ & $3.90,1.24$ & $3.90,1.27$ \\
$-1 \%$ & $3.99,1.29$ & $3.93,1.24$ & $3.95,1.25$ & $4.00,1.39$ & $3.95,1.21$ & $3.94,1.30$ & $3.93,1.25$ & $3.93,1.28$ & $3.93,1.25$ & $3.93,1.28$ \\
$0 \%$ & $3.98,1.29$ & $3.94,1.25$ & $3.93,1.24$ & $4.00,1.40$ & $3.97,1.22$ & $3.96,1.31$ & $3.95,1.21$ & $3.95,1.31$ & $3.95,1.21$ & $3.95,1.31$ \\
$1 \%$ & $4.00,1.32$ & $3.97,1.27$ & $3.96,1.26$ & $4.03,1.41$ & $4.00,1.22$ & $3.98,1.32$ & $3.96,1.27$ & $3.97,1.32$ & $3.96,1.27$ & $3.97,1.32$ \\
$2 \%$ & $4.00,1.32$ & $3.97,1.29$ & $3.96,1.27$ & $4.03,1.43$ & $4.00,1.22$ & $3.98,1.33$ & $3.97,1.28$ & $3.99,1.33$ & $3.98,1.28$ & $3.99,1.33$ \\
$3 \%$ & $3.99,1.32$ & $3.98,1.30$ & $3.96,1.27$ & $4.03,1.44$ & $3.99,1.22$ & $3.96,1.33$ & $3.96,1.29$ & $3.99,1.33$ & $3.96,1.29$ & $3.99,1.33$ \\
$4 \%$ & $3.96,1.33$ & $3.97,1.31$ & $3.95,1.28$ & $4.02,1.45$ & $3.97,1.22$ & $3.94,1.34$ & $3.94,1.28$ & $3.97,1.33$ & $3.95,1.29$ & $3.97,1.33$ \\
$5 \%$ & $3.93,1.33$ & $3.96,1.32$ & $3.92,1.29$ & $4.00,1.45$ & $3.95,1.22$ & $3.91,1.34$ & $3.91,1.28$ & $3.94,1.34$ & $3.91,1.28$ & $3.94,1.33$ \\
\hline \hline
\end{tabular}

under tensile strain. In the case of partial $\mathrm{Ca}$ substitution of $\mathrm{Sr}$ in $\mathrm{Sr}_{2} \mathrm{FeOsO}_{6}$ it has been argued that the structural transition from tetragonal to monoclinic symmetry is due to the fact that stronger/weaker lattice distortions support AFM/FM coupling [24]. Indeed, the induced chemical pressure drives a significant reduction of the $c / a$ ratio due to rotations of the oxygen octahedra around the [110] axis $\left(a^{-} a^{-} b^{+}\right)$[23]. Similarly, we find at $1 \%$ tensile strain that the reduction of the $c / a$ ratio is larger for the $P 2_{1} / n$ than the $I 4 / m$ symmetry by $1.2 \%$. This fact causes rotations of the oxygen octahedra around the [110] axis $\left(a^{-} a^{-} b^{+}\right)$, i.e., bending of the out-of-plane Fe-OOs bonds, and therefore out-of-plane AFM coupling. Together with the in-plane AFM coupling this results in FiM ordering.

To understand the evolution of the critical temperature under epitaxial strain, we next extract the magnetic coupling constants by mapping the energies obtained from density functional theory onto a Heisenberg model, $E=-\sum_{i j} J_{i j} \vec{S}_{i}$. $\vec{S}_{j}$, where $J_{i j}$ is the coupling constant between sites $i$ and $j$, and $\vec{S}_{i}$ and $\vec{S}_{j}$ are the spin vectors. It is necessary to include the nearest-neighbor (NN) and next-nearest-neighbor (NNN) exchanges to obtain the critical temperature in agreement with experiment [15]. We consider the coupling constants defined in Fig. 2, i.e., the in-plane NN exchange $\left(J_{1}\right)$, the out-of-plane NN exchange $\left(J_{2}\right)$, the in-plane NNN exchanges along the $a$ axis $\left(J_{3}\right)$ and $b$ axis $\left(J_{4}\right)$, and the two out-of-plane NNN exchanges $\left(J_{5}, J_{6}\right)$. When we assume that the spin vectors are collinear with magnitude 1 (as the real magnitude later will be taken into account in the Monte Carlo simulations), we can compute the magnetic coupling constants by solving the coupled equations

$$
\begin{aligned}
& E_{1}=E_{0}+8 J_{1}-4 J_{2}+4 J_{3}+4 J_{4}-8 J_{5}-8 J_{6}(\mathrm{~A}-\mathrm{AFM}), \\
& E_{2}=E_{0}-8 J_{1}+4 J_{2}+4 J_{3}+4 J_{4}-8 J_{5}-8 J_{6}(\mathrm{C}-\mathrm{AFM}), \\
& E_{3}=E_{0}-8 J_{1}-4 J_{2}+4 J_{3}+4 J_{4}+8 J_{5}+8 J_{6}(\mathrm{FiM}), \\
& E_{4}=E_{0}+8 J_{1}+4 J_{2}+4 J_{3}+4 J_{4}+8 J_{5}+8 J_{6}(\mathrm{FM}), \\
& E_{5}=E_{0}+4 J_{3}+4 J_{4}+8 J_{5}-8 J_{6} \\
& E_{6}=E_{0}+4 J_{3}+4 J_{4}-8 J_{5}+8 J_{6} \\
& E_{7}=E_{0}-4 J_{2}-4 J_{3}+4 J_{4} \\
& E_{8}=E_{0}+4 J_{2}-4 J_{3}+4 J_{4} \\
& E_{9}=E_{0}-4 J_{2}+4 J_{3}-4 J_{4} \\
& E_{10}=E_{0}+4 J_{2}+4 J_{3}-4 J_{4}
\end{aligned}
$$

where $E_{0}$ is the lattice energy and $E_{1}-E_{10}$ are the energies of the magnetic orderings obtained from density functional theory. The corresponding Fe and Os magnetic moments are summarized in Table I.

According to the results in Fig. 4 and in agreement with Ref. [24], we find without strain that the NN exchange is AFM and approximately four times stronger in the in-plane $\left(J_{1}\right)$ than in the out-of-plane $\left(J_{2}\right)$ direction. $J_{1}$ decreases/increases and $J_{2}$ increases/decreases under tensile/compressive strain due to the decreasing/increasing $c / a$ ratio and superexchange angle. Despite the AFM NN exchanges, the compound realizes below $0.8 \%$ tensile strain $\mathrm{C}$-AFM ordering due to the growing dominance of $J_{5}$ and $J_{6}$ combined over $J_{2}$. On the other hand, above $0.8 \%$ tensile strain ( $P 2_{1} / n$ symmetry) $J_{1}$ and $J_{2}$ exceed all other coupling constants due to favorable superexchange angles. This dominance of $J_{1}$ and $J_{2}$ reduces the spin frustration and leads to FiM ordering (resembling $\mathrm{Ca}_{2} \mathrm{FeOsO}_{6}$ [17]). Strain thus turns out to be an efficient tool for controlling the spin frustration.

To compute the critical temperature of the magnetic ordering for the different strain values we employ classical Monte Carlo simulations [31] for Ising spins in a $12 \times 12 \times 12$ supercell, using 80000 sweeps for thermalization and 70000 additional sweeps in the equilibrium. The obtained susceptibilities are shown in Fig. 5 and the resulting phase diagram

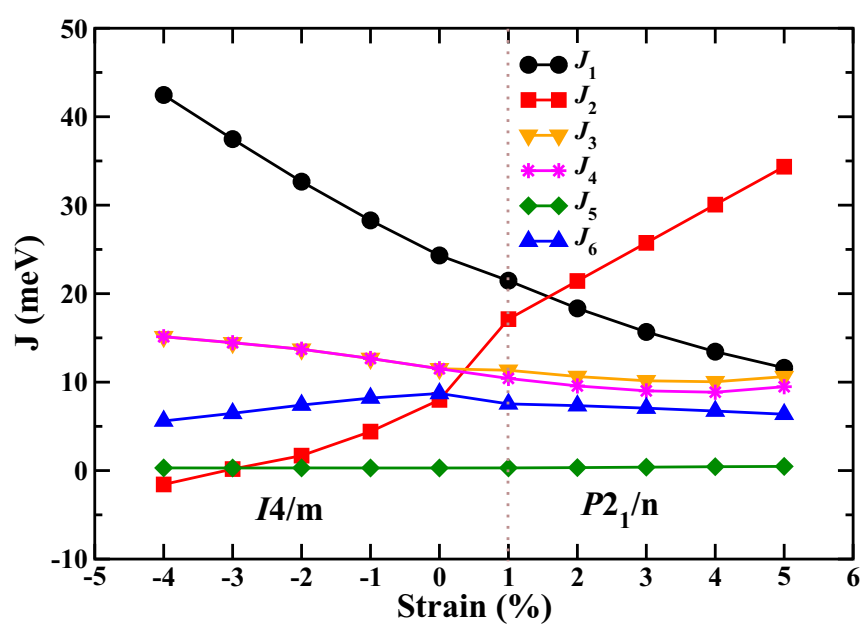

FIG. 4. Variation of the magnetic coupling constants under epitaxial strain. 

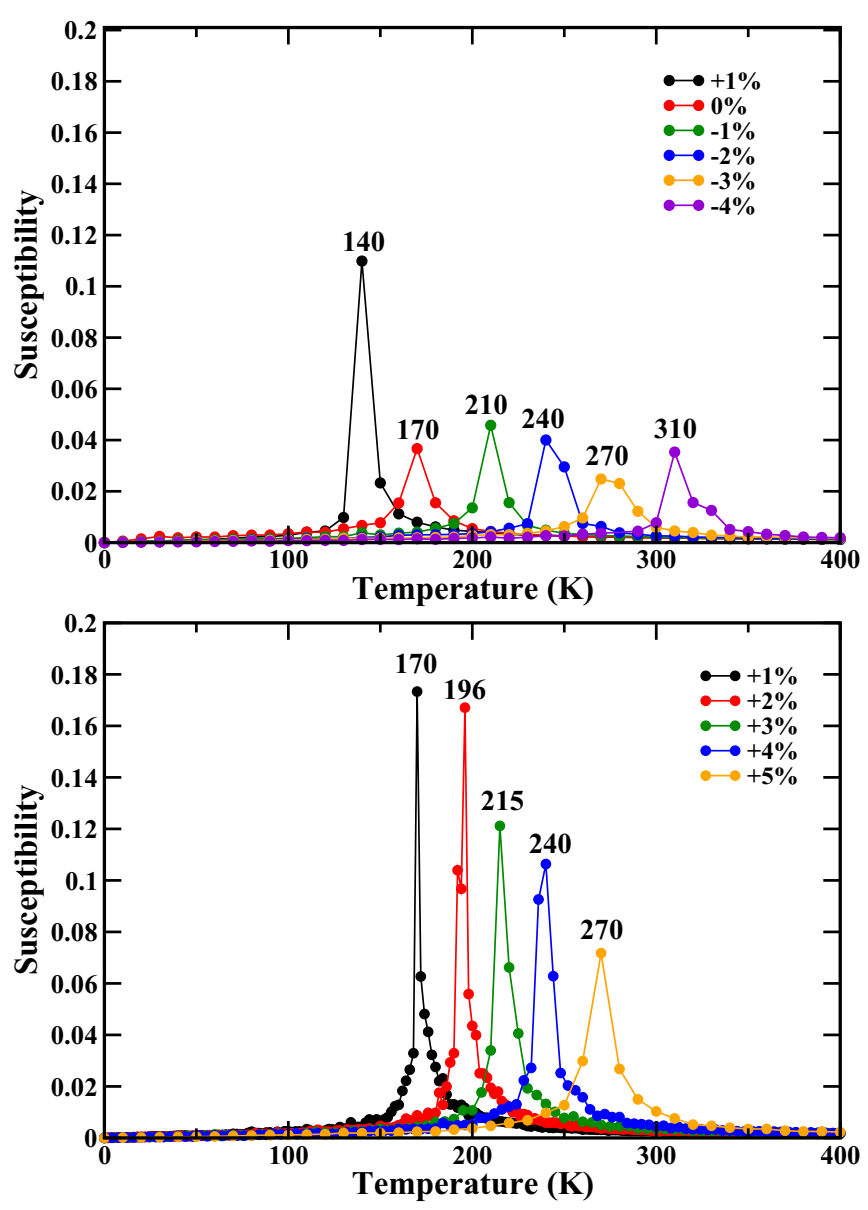

FIG. 5. Susceptibilities of the $I 4 / m$ (top) and $P 2_{1} / n$ (bottom) phases as functions of the temperature at various epitaxial strains. The peaks indicate the critical temperatures.

is given in Fig. 6. Without strain we find a low $T_{N}$ close to the experimental value of $140 \mathrm{~K}$, which has been attributed to competing magnetic interactions in Ref. [24]. Under compressive strain $J_{1}$ dominates more and more over the other

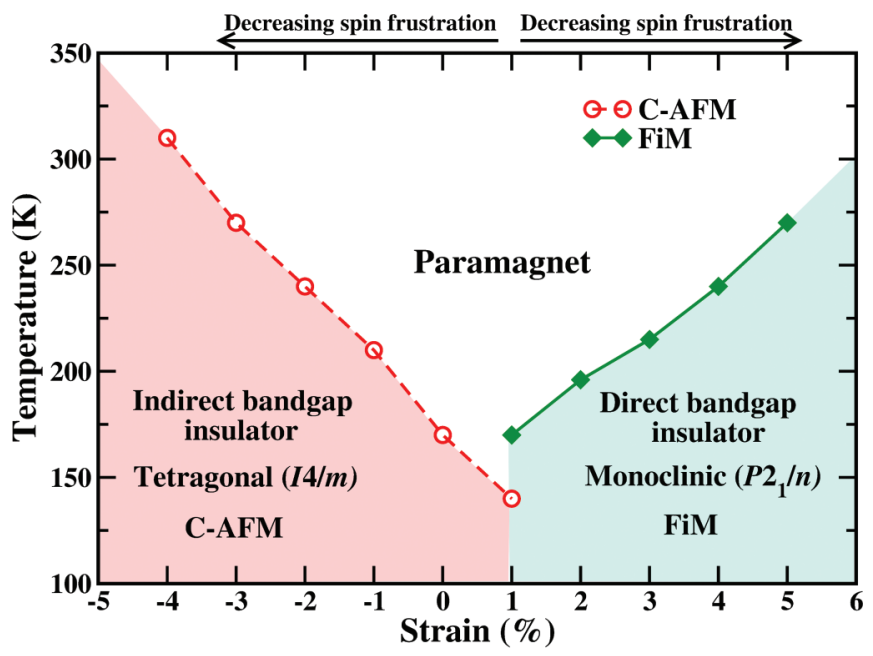

FIG. 6. Strain-temperature phase diagram obtained from Monte Carlo simulations.
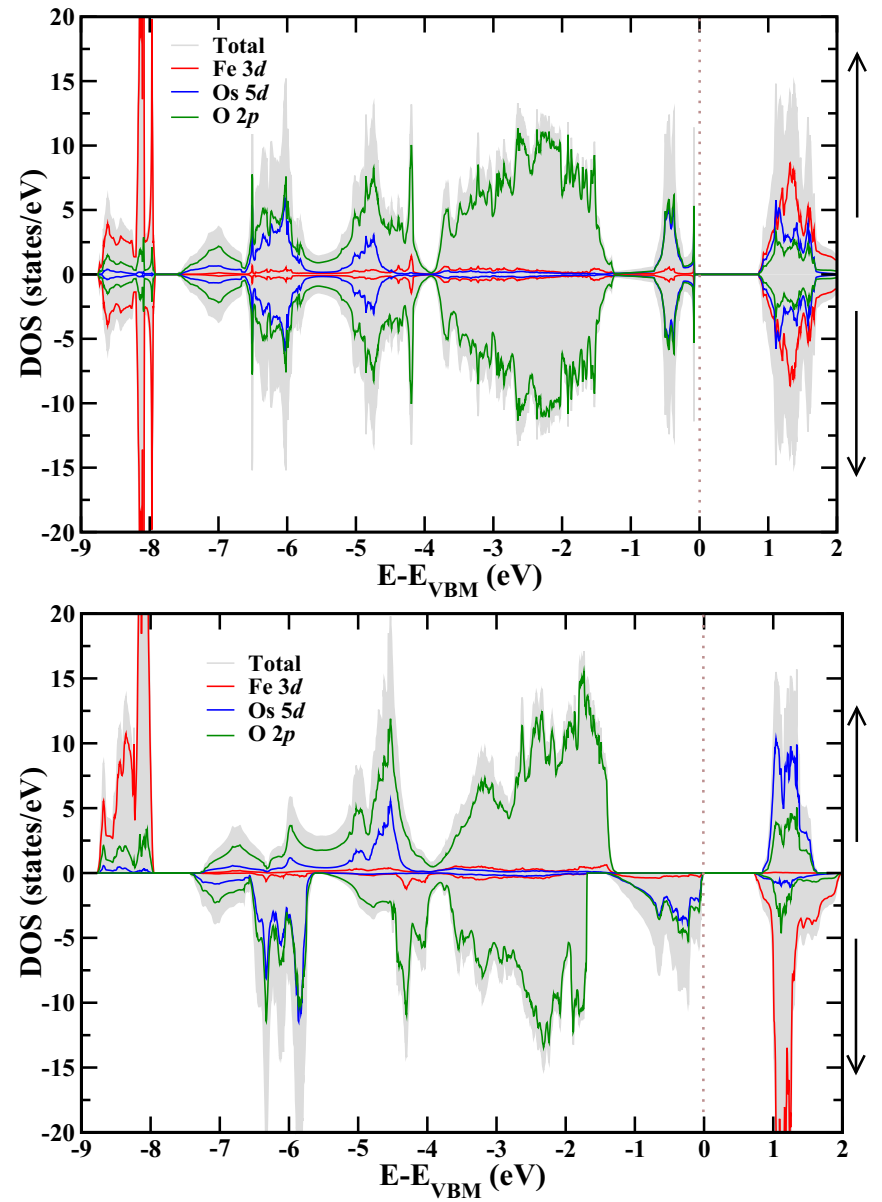

FIG. 7. DOS obtained for the C-AFM phase without strain (top) and the FiM phase at $4 \%$ tensile strain (bottom). Dotted vertical lines indicate the valence band maximum.

coupling constants (Fig. 4), which reduces the spin frustration. Thus, $T_{N}$ increases to $310 \mathrm{~K}$ at $-4 \%$ compressive strain. On the other hand, the Curie temperature $\left(T_{C}\right)$ of the FiM ordering increases to $270 \mathrm{~K}$ at $5 \%$ tensile strain. This behavior also can be attributed to a reduction of the spin frustration, as under tensile strain $J_{2}$ dominates more and more over the other coupling constants (Fig. 4).

According to Fig. 7, the up- and down-spin channels are equal without strain due to the C-AFM ordering. As mentioned earlier, effects of spin-orbit coupling can be neglected (Fig. 8). In the FiM phase the magnetic moments of NN $\mathrm{Fe}$ and $\mathrm{Os}$ atoms are aligned antiparallel in the in-plane and out-of-plane directions. Therefore, the up- and downspin channels are not equal and we obtain a net magnetic moment of $2 \mu_{B}$ per formula unit. Throughout the considered range of epitaxial strain the $\mathrm{Fe}$ and Os atoms maintain $3+\left(t_{2 g}^{3} e_{g}^{2}\right)$ and $5+\left(t_{2 g}^{3} e_{g}^{0}\right)$ oxidation states, respectively, with high spin configurations. Due to these oxidation states and the $158^{\circ}$ in-plane superexchange angle, we have always AFM ordering in the in-plane directions. Below $0.8 \%$ tensile strain we have FM ordering in the out-of-plane direction due to the $180^{\circ}$ out-of-plane superexchange angle [32-34], which gives rise to an overall C-AFM ordering. Above $0.8 \%$ tensile strain the out-of-plane $\mathrm{Fe}-\mathrm{O}-\mathrm{O}$ s bonds bend 


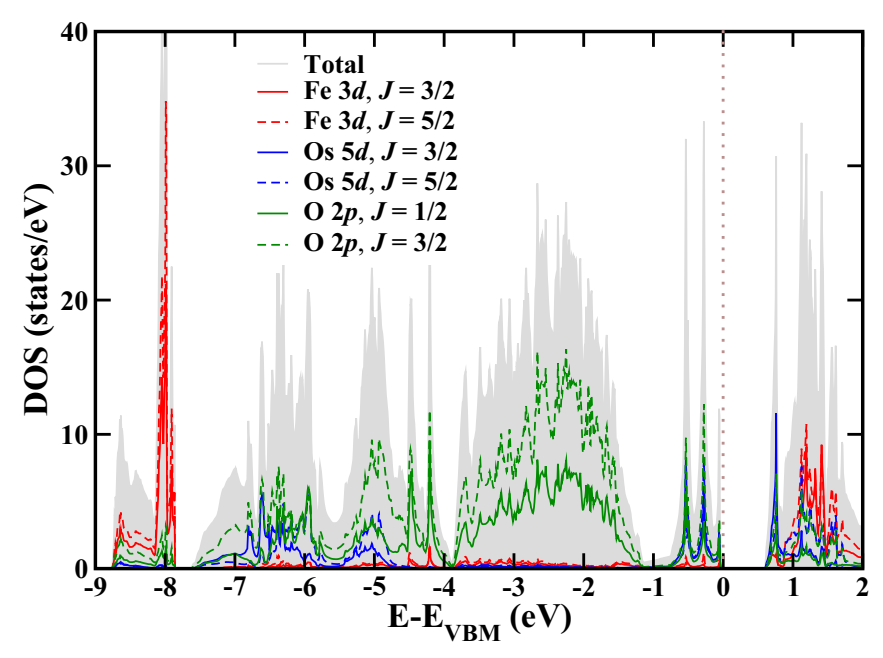

FIG. 8. DOS obtained for the C-AFM phase without strain, taking into account spin-orbit coupling.

significantly, leading to a $\sim 160^{\circ}$ out-of-plane superexchange angle and, therefore, AFM ordering in the out-of-plane direction, which gives rise to overall FiM ordering with a net magnetic moment of $2 \mu_{B}$ per formula unit. We find that the spin coherence length $\lambda_{c}=\pi /\left|k_{F}^{\uparrow}-k_{F}^{\downarrow}\right|$ of the FiM phase under $4 \%$ tensile strain $(2.96 \mathrm{~nm})$ clearly exceeds that of the leading $3 d-5 d$ room-temperature spintronics material $\mathrm{Sr}_{2} \mathrm{FeReO}_{6}$ $(2.16 \mathrm{~nm})$.

As shown in Fig. 9, we obtain a small band gap that further decreases both under compressive and tensile strain. The small band gap and the fact that the transition from $I 4 / \mathrm{m}$ to $P 2_{1} / n$ symmetry is accompanied by an indirect-to-direct band gap transition (Fig. 10) enables efficient absorption of sunlight. Figure 7 shows for the C-AFM phase that the valence band edge is dominated by hybridized Os $5 d$ and O $2 p$ states, whereas the conduction band edge additionally involves $\mathrm{Fe} d$ states. For the FiM phase the up-spin channel is characterized by a wide band gap of $2.2 \mathrm{eV}$ and the down-spin channel by a narrow band gap of $0.8 \mathrm{eV}$. In the down-spin channel (which forms the direct band gap) the valence band edge is dominated by hybridized Os $5 d$

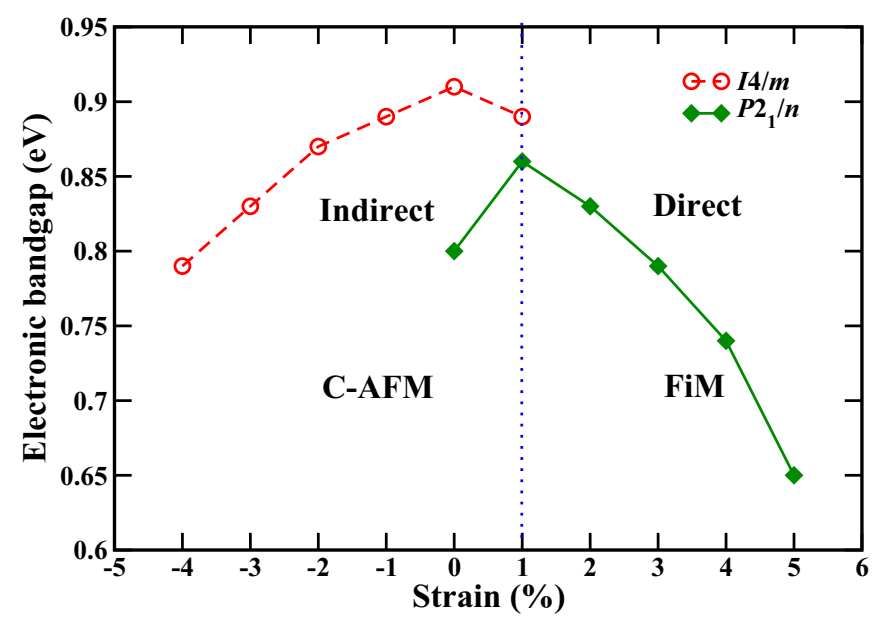

FIG. 9. Variation of the electronic band gap under epitaxial strain.

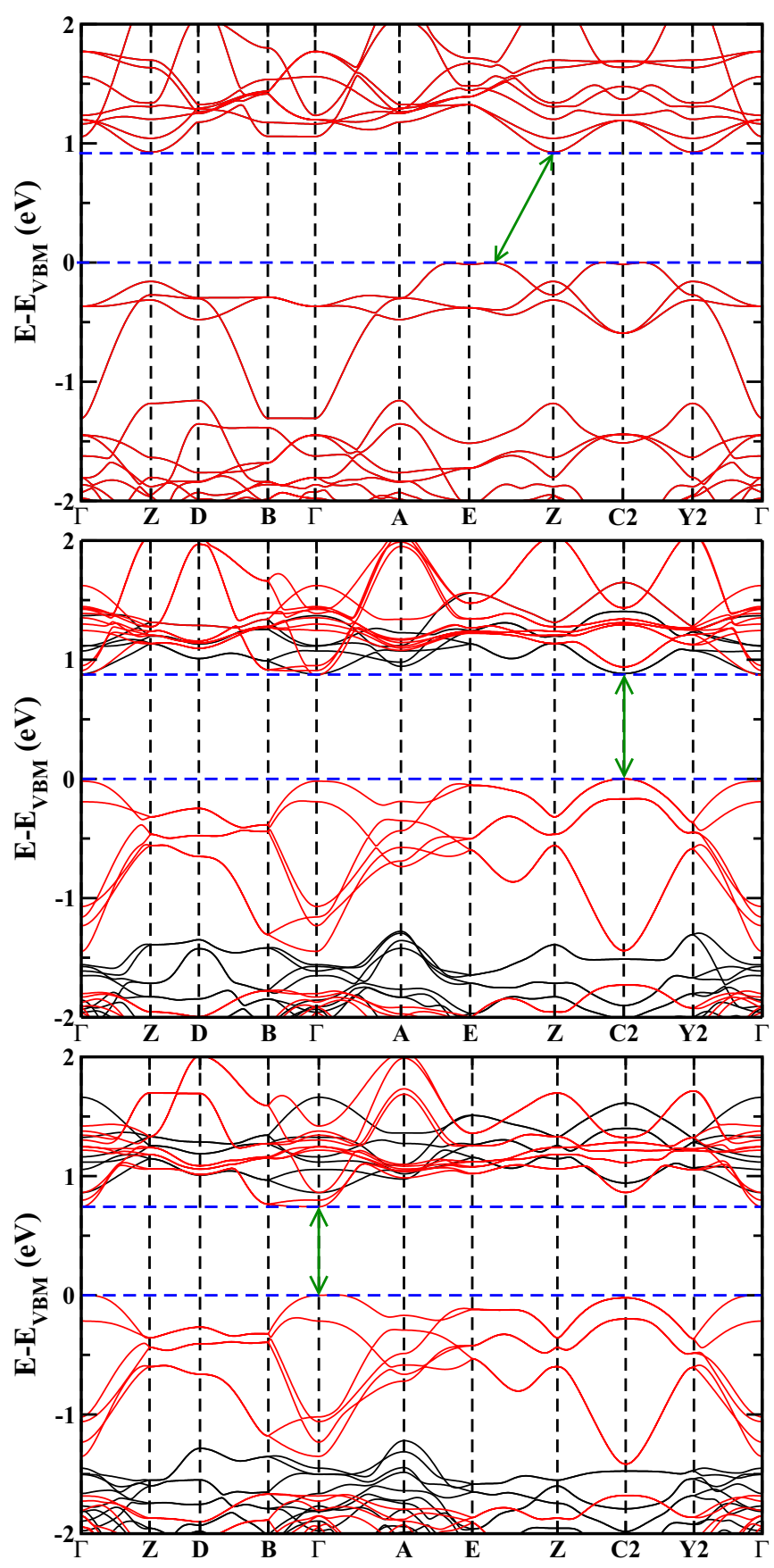

FIG. 10. Electronic band structure of the C-AFM phase without strain (top), FiM phase at $1 \%$ tensile strain (middle), and FiM phase at $4 \%$ tensile strain (bottom). Black/red lines represent the spinup/spin-down channel.

and $\mathrm{O} 2 p$ states, whereas the conduction band edge is almost entirely due to $\mathrm{Fe} 3 d$ states. As a consequence, photoexcitation will lead to a spatial electron-hole separation between the $\mathrm{Fe}$ (conduction band minimum) and Os (valence band maximum) sublattices, similar to $\mathrm{Bi}_{2} \mathrm{FeCrO}_{6}$ [35], implying that the electron-hole transition matrix element and thus the electron-hole recombination rate are small, which is desirable for photovoltaics. Based on the frequency-dependent complex dielectric function, we obtain for $\mathrm{Sr}_{2} \mathrm{FeOsO}_{6}$ an outstanding spectroscopic limited maximum efficiency [36] of $32 \%$, 
outperforming even the prototypical perovskite solar cell material $\mathrm{MAPbI}_{3}(\mathrm{MA}=$ methylammonium; $31 \%)$.

\section{CONCLUSION}

In conclusion, $\mathrm{Sr}_{2} \mathrm{FeOsO}_{6}$ is found to undergo under epitaxial strain a phase transition from $I 4 / m$ to $P 2_{1} / n$ symmetry and from C-AFM to FiM ordering. Magnetic coupling constants extracted from a Heisenberg model indicate decreasing spin frustration under both tensile and compressive strain, and Monte Carlo simulations accordingly demonstrate enhancement of the critical temperature. Specifically, $T_{N}$ of the C-AFM phase increases to $\sim 310 \mathrm{~K}$ at $-4 \%$ compressive strain and $T_{C}$ of the FiM phase increases to $\sim 270 \mathrm{~K}$ at $5 \%$ tensile strain due to the decreasing spin frustration. Therefore, the competition between magnetic interactions, the prime reason behind the low $T_{N}$ of the C-AFM phase without strain, can be controlled by heteroepitaxy. The complex interplay of tensile strain with the spin and orbital degrees of freedom establishes a FiM phase in $\mathrm{Sr}_{2} \mathrm{FeOsO}_{6}$ and makes the compound a high$T_{C} 3 d-5 d$ insulator with a large magnetic moment of $2 \mu_{B}$ per formula unit (as $\mathrm{Ca}_{2} \mathrm{FeOsO}_{6}$ ), which opens application potentials in spintronics [37-39] and magnetic storage devices [40-42]. The observation of an indirect-to-direct band gap transition under epitaxial strain and electron-hole separation between the $\mathrm{Fe}$ and $\mathrm{Os}$ sublattices is interesting for photovoltaics.

\section{ACKNOWLEDGMENTS}

The research reported in this publication was supported by funding from King Abdullah University of Science and Technology (KAUST). For computer time, this research used the resources of the Supercomputing Laboratory at KAUST.
[1] H. Kato, T. Okuda, Y. Okimoto, Y. Tomioka, Y. Takenoya, A. Ohkubo, M. Kawasaki, and Y. Tokura, Appl. Phys. Lett. 81, 328 (2002).

[2] J. B. Philipp, P. Majewski, L. Alff, A. Erb, R. Gross, T. Graf, M. S. Brandt, J. Simon, T. Walther, W. Mader, D. Topwal, and D. D. Sarma, Phys. Rev. B 68, 144431 (2003).

[3] M. Retuerto, M.-R. Li, P. W. Stephens, J. Sánchez-Benítez, X. Deng, G. Kotliar, M. C. Croft, A. Ignatov, D. Walker, and M. Greenblatt, Chem. Mater. 27, 4450 (2015).

[4] K. Samanta, P. Sanyal, and T. Saha-Dasgupta, Sci. Rep. 5, 15010 (2015).

[5] Y. Tomioka, T. Okuda, Y. Okimoto, R. Kumai, K.-I. Kobayashi, and Y. Tokura, Phys. Rev. B 61, 422 (2000).

[6] M.-R. Li, J. P. Hodges, M. Retuerto, Z. Deng, P. W. Stephens, M. C. Croft, X. Deng, G. Kotliar, J. Sánchez-Benítez, D. Walker, and M. Greenblatt, Chem. Mater. 28, 3148 (2016).

[7] H. L. Feng, S. Calder, M. P. Ghimire, Y.-H. Yuan, Y. Shirako, Y. Tsujimoto, Y. Matsushita, Z. Hu, C.-Y. Kuo, L. H. Tjeng, T.-W. Pi, Y.-L. Soo, J. He, M. Tanaka, Y. Katsuya, M. Richter, and K. Yamaura, Phys. Rev. B 94, 235158 (2016).

[8] T. Kim, M. Uehara, S. Cheong, and S. Lee, Appl. Phys. Lett. 74, 1737 (1999).

[9] H. L. Feng, P. Adler, M. Reehuis, W. Schnelle, P. Pattison, A. Hoser, C. Felser, and M. Jansen, Chem. Mater. 29, 886 (2017).

[10] A. Cho and J. Arthur, Prog. Solid State Chem. 10, 157 (1975).

[11] K.-I. Kobayashi, T. Kimura, H. Sawada, K. Terakura, and Y. Tokura, Nature (London) 395, 677 (1998).

[12] K.-I. Kobayashi, T. Kimura, Y. Tomioka, H. Sawada, K. Terakura, and Y. Tokura, Phys. Rev. B 59, 11159 (1999).

[13] Y. Krockenberger, K. Mogare, M. Reehuis, M. Tovar, M. Jansen, G. Vaitheeswaran, V. Kanchana, F. Bultmark, A. Delin, F. Wilhelm, A. Rogalev, A. Winkler, and L. Alff, Phys. Rev. B 75, 020404(R) (2007).

[14] M. Lorenz and M. S. Ramachandra Rao, J. Phys. D 47, 030301 (2013).

[15] A. K. Paul, M. Jansen, B. Yan, C. Felser, M. Reehuis, and P. M. Abdala, Inorg. Chem. 52, 6713 (2013).

[16] A. K. Paul, M. Reehuis, C. Felser, P. M. Abdala, and M. Jansen, Z. Anorg. Allg. Chem. 639, 2421 (2013).
[17] H. L. Feng, M. Arai, Y. Matsushita, Y. Tsujimoto, Y. Guo, C. I. Sathish, X. Wang, Y.-H. Yuan, M. Tanaka, and K. Yamaura, J. Am. Chem. Soc. 136, 3326 (2014).

[18] R. Nechache, W. Huang, S. Li, and F. Rosei, Nanoscale 8, 3237 (2016).

[19] A. E. Taylor, R. Morrow, M. D. Lumsden, S. Calder, M. H. Upton, A. I. Kolesnikov, M. B. Stone, R. S. Fishman, A. Paramekanti, P. M. Woodward, and A. D. Christianson, Phys. Rev. B 98, 214422 (2018).

[20] A. K. Paul, M. Reehuis, V. Ksenofontov, B. Yan, A. Hoser, D. M. Többens, P. M. Abdala, P. Adler, M. Jansen, and C. Felser, Phys. Rev. Lett. 111, 167205 (2013).

[21] S. Kanungo, B. Yan, M. Jansen, and C. Felser, Phys. Rev. B 89, 214414 (2014).

[22] R. Morrow, J. W. Freeland, and P. M. Woodward, Inorg. Chem. 53, 7983 (2014).

[23] L. S. I. Veiga, G. Fabbris, M. van Veenendaal, N. M. SouzaNeto, H. L. Feng, K. Yamaura, and D. Haskel, Phys. Rev. B 91, 235135 (2015).

[24] Y. Hou, H. Xiang, and X. Gong, Sci. Rep. 5, 13159 (2015).

[25] X.-Y. Dong, S. Kanungo, B. Yan, and C.-X. Liu, Phys. Rev. B 94, 245135 (2016).

[26] P. Giannozzi, S. Baroni, N. Bonini, M. Calandra, R. Car, C. Cavazzoni, D. Ceresoli, G. L. Chiarotti, M. Cococcioni, I. Dabo, A. D. Corso, S. de Gironcoli, S. Fabris, G. Fratesi, R. Gebauer, U. Gerstmann, C. Gougoussis, A. Kokalj, M. Lazzeri, L. Martin-Samos et al., J. Phys.: Condens. Matter 21, 395502 (2009).

[27] V. I. Anisimov, F. Aryasetiawan, and A. I. Liechtenstein, J. Phys.: Condens. Matter 9, 767 (1997).

[28] A. Khare, A. Singh, S. S. Prabhu, and D. S. Rana, Appl. Phys. Lett. 102, 192911 (2013).

[29] G. Vinai, A. Khare, D. S. Rana, E. D. Gennaro, B. Gobaut, R. Moroni, A. Y. Petrov, U. Scotti di Uccio, G. Rossi, F. Miletto Granozio, G. Panaccione, and P. Torelli, APL Mater. 3, 116107 (2015).

[30] N. Ganguli and P. J. Kelly, Phys. Rev. Lett. 113, 127201 (2014).

[31] C. Escorihuela-Sayalero, O. Diéguez, and J. Íñiguez, Phys. Rev. Lett. 109, 247202 (2012). 
[32] J. B. Goodenough, Phys. Rev. 100, 564 (1955).

[33] J. Kanamori, J. Phys. Chem. Solids 10, 87 (1959).

[34] E. O. Wollan, Phys. Rev. 117, 387 (1960).

[35] D. Kim, H. Han, J. H. Lee, J. W. Choi, J. C. Grossman, H. M. Jang, and D. Kim, Proc. Natl. Acad. Sci. U.S.A. 115, 6566 (2018).

[36] L. Yu and A. Zunger, Phys. Rev. Lett. 108, 068701 (2012).

[37] Y. Yamada, K. Ueno, T. Fukumura, H. Yuan, H. Shimotani, Y. Iwasa, L. Gu, S. Tsukimoto, Y. Ikuhara, and M. Kawasaki, Science 332, 1065 (2011).
[38] X. Li, X. Wu, Z. Li, and J. Yang, Phys. Rev. B 92, 125202 (2015).

[39] J. Xu, C. Xu, J.-B. Liu, L. Bellaiche, H. Xiang, B.-X. Liu, and B. Huang, npj Comput. Mater. 5, 114 (2019).

[40] P. Sharma, A. Gupta, K. Rao, F. J. Owens, R. Sharma, R. Ahuja, J. O. Guillen, B. Johansson, and G. Gehring, Nat. Mater. 2, 673 (2003).

[41] K. Ando, Science 312, 1883 (2006).

[42] W. Liu, H. Zhang, J.-A. Shi, Z. Wang, C. Song, X. Wang, S. Lu, X. Zhou, L. Gu, D. V. Louzguine-Luzgin et al., Nat. Commun. 7, 13497 (2016). 